\title{
Article
}

\section{Gait Phase Estimation Based on User-Walker Interaction Force}

\author{
Pengcheng Li ${ }^{1, *}$, Yasuhiro Akiyama ${ }^{1} \mathbb{C}$, Xianglong Wan ${ }^{2}$, Kazunori Yamada ${ }^{3}$, Mayu Yokoya ${ }^{3}$ and Yoji Yamada ${ }^{1}$ \\ 1 Department of Mechanical System Engineering, Nagoya University, Furo-cho, Chikusa-ku, \\ Nagoya 464-8603, Japan; akiyama-yasuhiro@mech.nagoya-u.ac.jp (Y.A.); \\ yoji.yamada@mae.nagoya-u.ac.jp (Y.Y.) \\ 2 School of Automation and Electrical Engineering, University of Science and Technology Beijing, \\ 30 Xueyuan Road, Haidian District, Beijing 100083, China; wanxianglong@ustb.edu.cn \\ 3 Business Innovation Division, Panasonic Corp., Oaza Kadoma, Kadoma-shi, Osaka 571-8501, Japan; \\ yamada.kazunori@jp.panasonic.com (K.Y.); yokoya.mayu@jp.panasonic.com (M.Y.) \\ * Correspondence: li.pengcheng@g.mbox.nagoya-u.ac.jp
}

check for updates

Citation: Li, P.; Akiyama, Y.; Wan, X.; Yamada, K.; Yokoya, M.; Yamada, Y. Gait Phase Estimation Based on User-Walker Interaction Force. Appl. Sci. 2021, 11, 7888. https://doi.org/ 10.3390/app11177888

Academic Editor: Subhas Mukhopadhyay

Received: 11 June 2021

Accepted: 20 August 2021

Published: 26 August 2021

Publisher's Note: MDPI stays neutral with regard to jurisdictional claims in published maps and institutional affiliations.

Copyright: (c) 2021 by the authors. Licensee MDPI, Basel, Switzerland. This article is an open access article distributed under the terms and conditions of the Creative Commons Attribution (CC BY) license (https:// creativecommons.org/licenses/by/ $4.0 /)$.

\begin{abstract}
Smart walkers have been developed for assistance and rehabilitation of elderly people and patients with physical health conditions. A force sensor mounted under the handle is widely used in smart walkers to establish a human-machine interface. The interaction force can be used to control the walker and estimate gait parameters using methods such as the Kalman filter for real-time estimation. However, the estimation performance decreases when the peaks of the interaction force are not captured. To improve the stability and accuracy of gait parameter estimation, we propose an online estimation method to continuously estimate the gait phase and cadence. A multiple model switching mechanism is introduced to improve the estimation performance when gait is asymmetric, and an adaptive rule is proposed to improve the estimation robustness and accuracy. Simulations and experiments demonstrate the effectiveness and accuracy of the proposed gait parameter estimation method. Here, the average estimation error for the gait phase is $0.691 \mathrm{rad}$ when the gait is symmetric and $0.722 \mathrm{rad}$ when it is asymmetric.
\end{abstract}

Keywords: smart walker; gait phase estimation; adaptive control; switching mechanism

\section{Introduction}

With aging, muscle strength, cognition, and coordination gradually deteriorate, possibly leading to loss of mobility [1]. Physical therapists advise elderly people to stay physically active to preserve or restore mobility [2,3]. Walkers are widely prescribed by clinicians to such patients to enlarge the base of support for improved walking stability [4]. Furthermore, robotic walkers are wheeled walkers equipped with motors and sensors that can be used to assist physical therapists in rehabilitation treatment to help elderly people regain mobility [5].

To achieve proper rehabilitation using robotic walkers, it is essential to obtain gait parameters, such as the instants of heel strike (HS) and toe-off, cadence, and gait phase. The gait parameters captured during walking can be used for the gait ability assessment continuously so that the clinicians can monitoring the rehabilitation process and adjust the training plans accordingly [6]. In addition, gait parameters can be used to identify abnormal gait patterns for providing more suitable assistance to users [7]. From the sensors embedded in walkers, the force sensor under the handle has been the most important given its simplicity and reliability. Additionally, force sensors do not require additional environment or user preparation. Alwan [8,9] proposed an approach to assess gait parameters based on the user-walker interaction force. The peak timing of the vertical components of the interaction force on one side of the handle coincided with the instant of ipsilateral HS. A similar correlation between force and gait parameters was adopted by Abellanas [10], who used the sum of the interaction force detected by two sensors mounted on the two handles to detect the HS instant. However, no method has been devised for real-time gait 
phase estimation, which is crucial for developing control methods for rehabilitation. In fact, obtaining and analyzing gait phase information can help develop rehabilitation strategies. For example, function-related muscles can be strengthened at the precise timing as when the corresponding muscle groups are activated, as demonstrated in a previous study, in which we proposed gait-phase-dependent control for rehabilitation [11].

To provide proper assistance and maximize the rehabilitation outcomes, various assistive robots and rehabilitation robots extract information of the user's gait phases during walking [12]. Such information is essential for wearable robots because if they cannot provide the proper assistance in the correct gait phases; they would be a burden rather than a support. Lewis [13] used the instants of HS detected from foot switches and the average duration of the last several gait cycles to estimate the current gait phase. Hence, gait phase of a walker user can be estimated by leveraging Abellanas's method, where the gait phase can be considered as the accumulation of the estimated frequency that starts from an HS. Researchers have applied the cycles of kinetic or kinematic data of ground reaction force or joint angle for estimating the gait phase by using adaptive oscillators (AOs) $[14,15]$. An AO is a mathematical tool proposed by Righetti $[16,17]$ that synchronizes its phase and frequency with any quasi-periodic signal to extract these parameters from the signal.

Despite its accurate gait events estimation, the Abellanas's method presents some limitations. (1) When it is directly applied to estimate the gait phase, the estimation accuracy depends on the correct detection of gait events and predicted duration of the gait cycle, which is in turn based on previous gait cycle durations [18]. (2) The estimation performance deteriorates when gait event detection fails because an incorrect duration degrades the estimation accuracy of the gait phase in the subsequent cycles, and the estimated phase is the integral of the estimated frequency. If a considerable phase estimation error occurs, it can only diminish when the next gait cycle starts, leading to discontinuity in phase estimation during the transition period. The $\mathrm{AO}$ can provide a zero-delay accurate frequency and phase estimation provided that the input signal has slow dynamics. However, a drastic change in the frequency or amplitude of the target signal substantially degrades the estimation accuracy due to slow convergence. Although the estimation convergence can be accelerated by increasing the gains in the estimation loop, fluctuations in the estimates may occur $[19,20]$. As for the case of interaction force based gait phase estimation, the amplitude of the interaction force is not constant but can change drastically because it depends on the bodyweight transmitted to the handle. In addition, although the frequency of walking does not change rapidly, many people exhibit asymmetric gait patterns [21], which may vary the signal frequency over consecutive gait cycles. Therefore, it is crucial to mitigate the influence of the transient changes to reach an accurate and stable gait phase estimation.

In this paper, we present a real-time method to estimate the gait phases based on the user-walker interaction force. An AO extracts the frequency and phase information from the oscillatory gait component. To improve the performance for users with gait impairments, we also propose a switching mechanism and an adaptive rule for the estimation parameters. The switching mechanism can switch between two adaptive oscillators so that the walking frequencies of two consecutive gait cycles can be tracked. The adaptive rule changes the parameter of the $\mathrm{AO}$ to mitigate the influence of the transient change in the amplitude of the interaction force in gait phase estimation.

\section{Method for Asymmetric Gait Phase Estimation}

In this section, the method proposed for gait phase estimation is described. In Section 2.1, the architecture of the estimation method is introduced. In Sections 2.2 and 2.3, we briefly introduce the methods adopted for the gait phase estimation. The proposed adaptive methods for the robust estimation are demonstrated in Section 2.4. 


\subsection{Architecture}

When a user walks with a walker, its handle supports part of the bodyweight of the user to reduce the physical burden to the lower limbs by transferring force to the upper limbs. The change in vertical position of the center of mass of a user during walking leads to a cyclic change in the interaction force in each gait cycle, as shown in Figure 1. In other words, the interaction force contains information of the gait phases. Frizera Neto [22] divided the interaction force into three components: (1) supporting force, which represents the part of the bodyweight that is placed on the walker; (2) slow oscillation caused by the cyclic change in the placement of the center of mass changing with the gait phase; and (3) high-frequency vibration originating from the walker. As the slow oscillation contains the information of the gait phase, we use it for estimation.

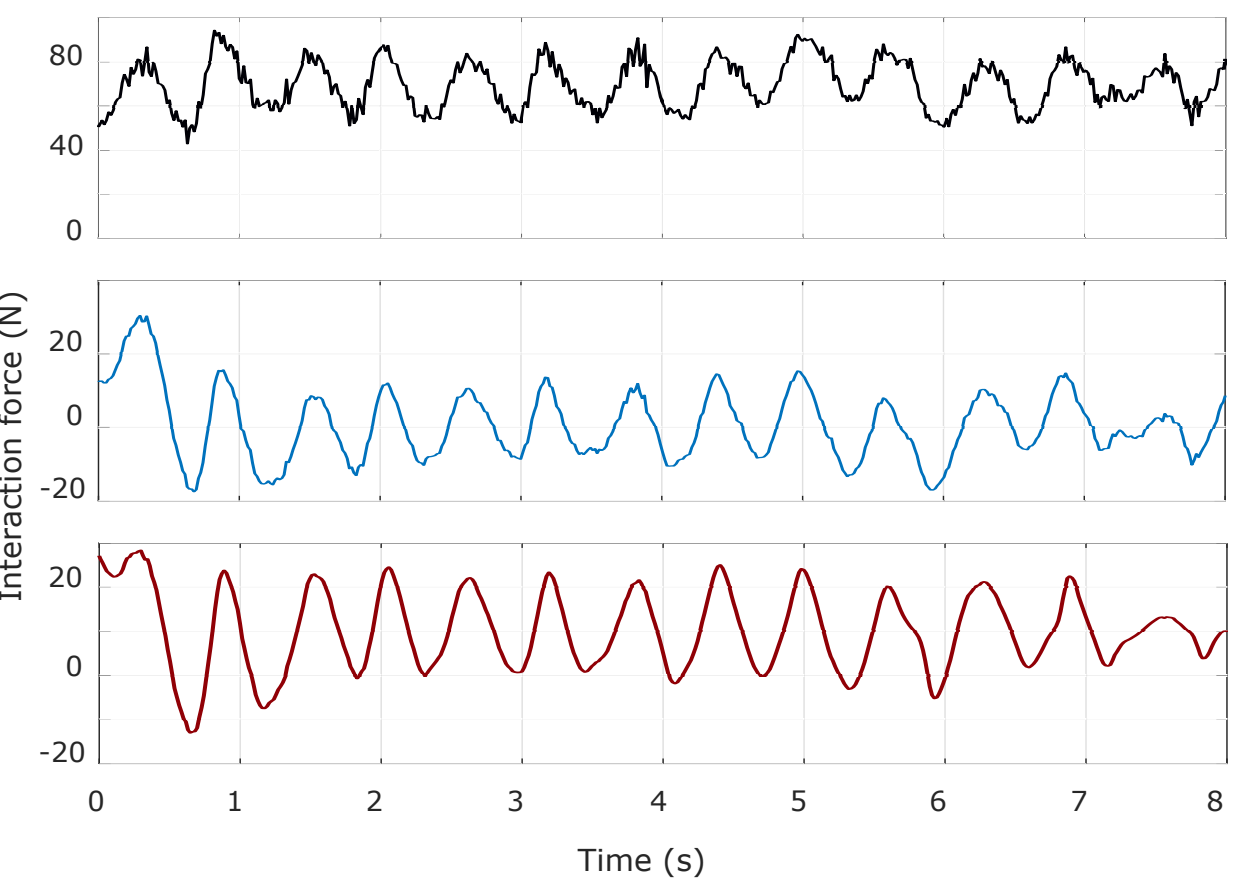

Figure 1. Interaction force along vertical direction. Black line represents the raw data of the interaction force, blue line represents the signal after BMFLC, and red line represents the reconstructed signal of the AOs.

The main tool used for gait phase estimation is the AO proposed by Righetti [16]. Nevertheless, the supporting force and high-frequency vibration in the user-walker interaction force degrade the estimation accuracy because the $\mathrm{AO}$ is sensitive to noise and variations in the input signal. Therefore, preprocessing is necessary to extract the low-frequency gait oscillations from raw data. As shown in Figure 2, the proposed estimation method consists of two subsystems. (1) A band-limited multiple Fourier linear combiner (BMFLC) [23,24] model extracts the slow gait oscillation from the raw data of the user-walker interaction force, enabling the AO to reflect the information of the gait phase. (2) Two AOs are used to separately obtain the gait phase information from the two legs. The difference between the slow oscillation and the $\mathrm{AO}$ output is used as feedback to tune its frequency and phase. In addition, alternate switching between the two AOs is applied in response to the input signal when the HS is detected. Moreover, an adaptive rule for the AO parameter is applied to reduce the impact of the transient amplitude change on phase estimation. 


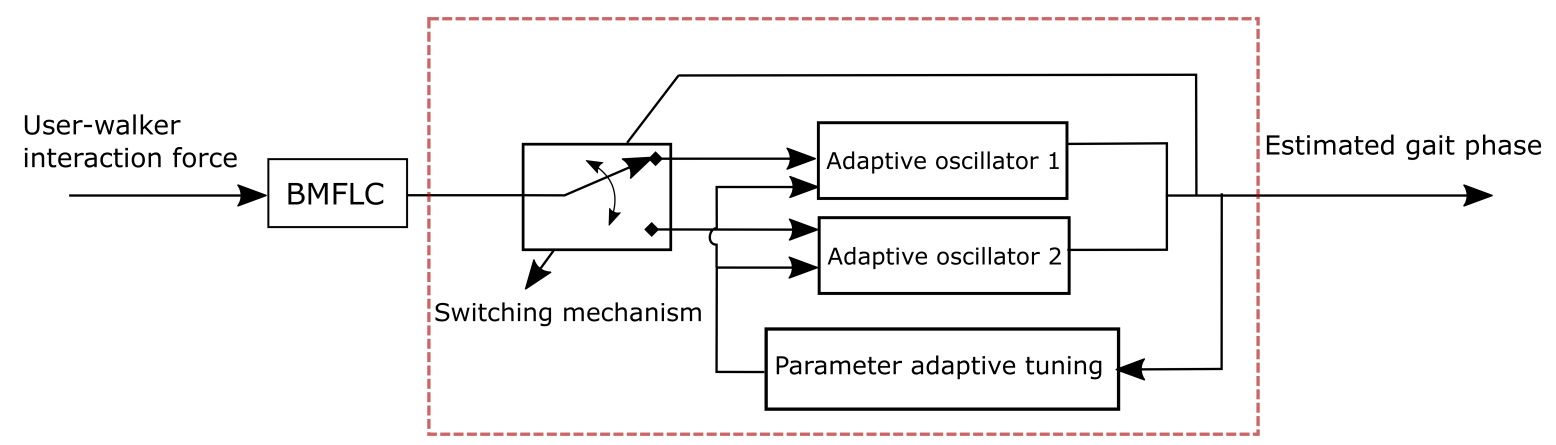

Figure 2. Architecture of proposed gait phase estimation method.

\subsection{BMFLC}

To separate gait phase frequency components from raw interaction force data, we adopt a model to reconstruct the interaction force by using BMFLC to represent the slow oscillation components in frequencies from $\gamma_{1}$ to $\gamma_{m}$, similar to the proposal by Velovulo [23]. Moreover, we use a constant component $P_{c}(k T)$ to represent the voluntary force.

$$
\begin{gathered}
P_{o}(k T)=\sum_{n=1}^{m}\left(a_{n}(k T) \times \sin \left(\gamma_{n}(k T)\right)+b_{n}(k T) \times \cos \left(\gamma_{n}(k T)\right)\right) \\
P_{c}(k T)=a_{0}(k T)
\end{gathered}
$$

where $T \in R$ is the sampling interval, $a_{0}$ is the voluntary force component, $a_{1}, b_{1}, \cdots, a_{m}, b_{m}$ represent the amplitudes of the sinusoidal signal components, $\Delta \gamma$ is the step size in frequency band $\left[\gamma_{1}-\gamma_{m}\right]$ given by $\Delta \gamma=\left(\gamma_{m}-\gamma_{1}\right) / n, P_{o}(k T)$ is the slow oscillation component, and $P_{c}(k T)$ is the constant component.

\section{3. $A O$}

The AOs developed by Righetti [16] synchronize with periodic signals and track the frequency and phase of the target signal without delay. The estimated parameters were used to reconstruct an approximate signal. The difference between the estimated and target signals can serve as feedback to adjust the states of the $\mathrm{AO}$ to adapt to changes in the target signal. An AO can be described by the following formulas:

$$
\begin{gathered}
\dot{r}=\left(\mu-r^{2}\right) \times r+\sigma \times F \times \cos \varphi \\
\dot{\varphi}=\omega-\frac{\sigma}{r} \times F \times \sin \varphi \\
\dot{\omega}=-\epsilon \times F \times \sin \varphi \\
\dot{\alpha}=\delta \times F \times r \times \cos \varphi \\
y=\alpha \times r \times \cos \varphi
\end{gathered}
$$

where $r, \varphi$, and $\omega$ are the amplitude, phase and frequency of the oscillator, respectively. $\alpha \times r$ is the amplitude of the reconstructed signal. $F$ is the difference between the reconstructed signal and target signal. $y$ is the reconstructed signal by the AO. The learning speed is adjusted by the constants of $\mu, \epsilon, \sigma$, and $\delta$. 


\subsection{Adaptive Rules for Transient Parameters}

During walking, gait phases increase with monotony and continuity owing to their nature. In $\mathrm{AO}$, however, the estimated phase, which is an $\mathrm{AO}$ parameter, does not necessarily always increase. Because the phase, frequency, and amplitude are estimated in the same loop, the phase may fluctuate owing to sudden changes in frequency or amplitude. Therefore, we propose two methods to handle phase fluctuations caused by sudden changes in frequency and amplitude, as detailed below.

\subsubsection{Switching Mechanism}

Robotic walkers are designed for users who require assistance or rehabilitation to enhance their physical abilities. Many people suffer from neuromuscular impairments or muscle atrophies, which lead to asymmetric gait patterns [21]. Thus, consecutive steps can notably vary in timing, hindering gait phase estimation using one AO.

In the 1990s, various control approaches with model switching were introduced to improve the performance of adaptive control under large and rapid changes in the system dynamics [25,26]. In particular, Westervelt [27] proposed switching between different controllers for a plantar biped walker to achieve high control stability under different cadences. Similarly, we adopt a simple switching mechanism for AOs to track rapid changes in the walking frequency. Specifically, two AOs with the same structure work in parallel, but only one is active at any instant. As the states of $r, \varphi, \alpha$ are shared between the AOs, their values are used by the active AO. On the other hand, there are two independent frequencies, $\omega_{1}$ and $\omega_{2}$, for the two AOs corresponding to the two legs.

During estimation, the value of phase $\varphi(t)$ monotonically increases. A modulo operation is applied to the gait phase estimated by the AOs:

$$
C_{\text {step }}=\bmod (\varphi(t), 2 \pi)
$$

Switching occurs when $C_{\text {step }}$ reaches $2 \pi$, and the gait characteristics of two consecutive steps are estimated by two different AOs. Thus, a sudden change in frequency caused by an asymmetric gait pattern between legs is considered by the proposed switching mechanism.

For some users, there is a substantial difference between the two AOs in amplitude. However, the amplitudes of both AOs vary substantially during walking. The improvement in estimation accuracy from using two independent amplitudes for AOs is limited. Furthermore, two AOs switch when the reconstructed signal $y$ is at its peak; therefore, the switch in amplitude will lead to a sudden change in the waveform of the reconstructed signal in a discontinuous manner. It is a perturbation to the AOs, which will eventually degrade the accuracy of phase estimation. We can choose a large $\delta$ which can scale the converge rate of $\alpha$, so that the $\mathrm{AO}$ can achieve a higher estimation accuracy when the amplitude of the input signal changes rapidly.

\subsubsection{Adaptive Rule for a Parameter}

The component of the interaction force used for gait phase estimation is the slower oscillation that reflects walking, whose amplitude can vary with the walking posture or elbow movements. The phase dynamics of the $\mathrm{AO}$ can be expressed as follows:

$$
\dot{\varphi}=\omega-\frac{\sigma}{r} \times F \times \sin \varphi
$$

when the residual $F$ is large enough and $F \times \sin \varphi>0$,

$$
\dot{\varphi}=\omega-\frac{\sigma}{r} \times F \times \sin \varphi<0
$$

Although the AO phase is supposed to increase monotonically, the estimated phase decreases when the condition above is satisfied. 
To prevent this problem, feedback gain $\sigma$ can be reduced for $\dot{\varphi}$ to be positive when the transient change occurs, thus limiting fluctuations in the estimated phase. However, a small $\sigma$ increases the time taken by the oscillator to synchronize with the input signal. This not only influences the estimation accuracy initially but also limits the ability to track gait changes [20]. Alternatively, we apply an adaptive rule for $\sigma$ when a sudden change in amplitude occurs.

A phase fluctuation can be identified by observing the difference between $\dot{\varphi}$ and $\omega$. For a small difference, $\sigma$ stays at its initial value. For a large difference, we replace $\sigma$ with $\bar{\sigma}$ as follows:

$$
\bar{\sigma}=\lambda \times \frac{(r \times \omega)}{F \times \sin \varphi}
$$

where $\lambda$ is a positive constant less than $1 . \dot{\varphi}$ is equal to $(1-\lambda) \times \omega$, guaranteeing that the phase changes almost as fast as the frequency. As a transient phase change is uncommon during walking, we consider a large phase change as a disturbance in the system dynamics. Therefore, we change the parameters to minimize the influence of such a disturbance. In this study, the difference between $\dot{\varphi}$ and $\omega$ is considered large when it is larger than $30 \%$ of $\omega$, which means the adaptive rule is applied when the change rate of phase is lower than $70 \%$ of the estimated frequency. As a result, a transient change can be bounded to improve the robustness against the disturbance and achieve smooth gait phase estimation. In addition, fast synchronization can be achieved when the difference in phase change is small, allowing synchronization to be maintained during walking and promoting accurate gait phase estimation.

\section{Simulation}

We conducted simulations to evaluate the influence of the asymmetric gait patterns and varying amplitudes of the input signal on gait phase estimation.

\subsection{Simulation Scenario 1}

Many people suffer from strokes and often experience conditions such as loss of coordination and hemiplegia afterward, resulting in asymmetric gait patterns [21]. The interaction force is naturally different between steps performed with a healthy leg and with a paralyzed leg. Such difference poses a challenge for gait phase estimation using AO because two frequencies occur alternately in the input signal. We used the simulated signal shown in Figure 3 to evaluate the switching scheme under asymmetric gait. The signal is sinusoidal with a constant amplitude and frequencies of $\omega_{s 1}$ and $\omega_{s 2}$ for consecutive cycles.

Figure 3 shows the estimation results when using a single $\mathrm{AO}$ and two AOs with the switching mechanism. For a single $\mathrm{AO}$, the estimated frequency is close to the mean frequency. The two AOs with the switching mechanism can reflect the two frequencies. The phase estimation error when using the single AO is clearly higher than that of the proposed method and increases with an increase in the difference between $\omega_{s 1}$ and $\omega_{s 2}$. The proposed estimation method with the switching mechanism maintains a small error, which is mostly attributable to the inaccurate switching instant that often occurs in a discrete system. Nevertheless, the error due to switching is small and can be further reduced by increasing the sampling rate.

The estimation error of the proposed method is caused by the poor frequency estimation accuracy given the slow convergence. The $\mathrm{AO}$ convergence rate for frequency estimation can be increased by tuning parameter $\epsilon$, but a larger $\epsilon$ increases the steady-state error, as also noted in $[18,19]$. 

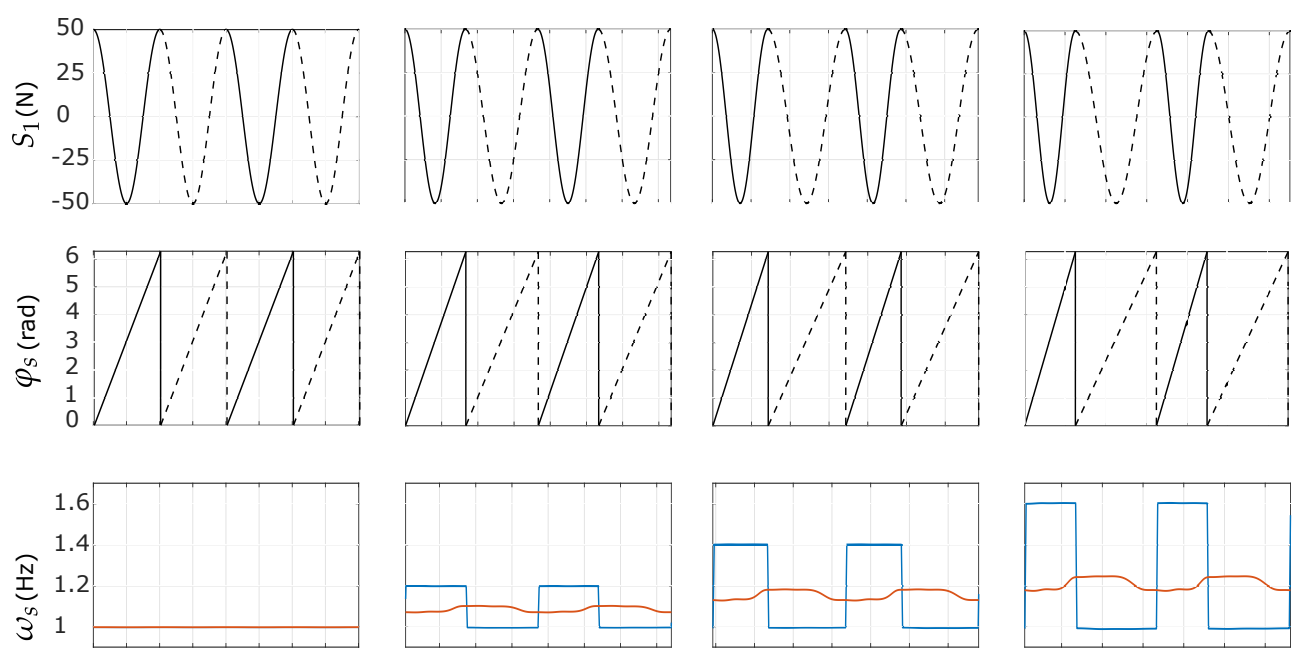

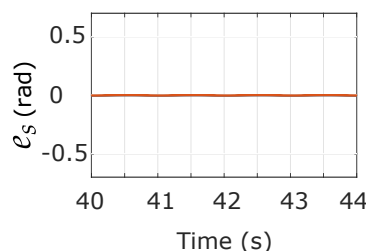

(a)
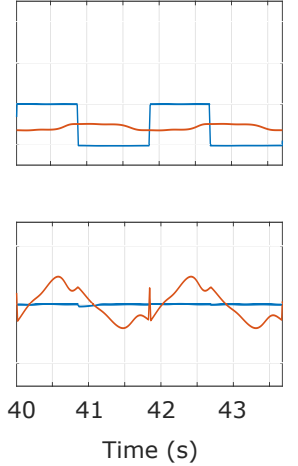

(b)
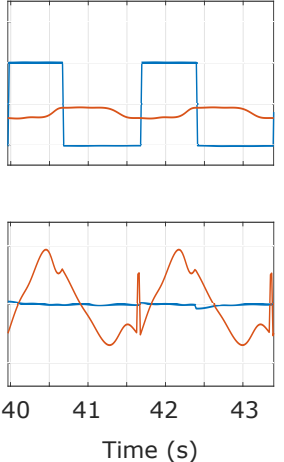

(c)

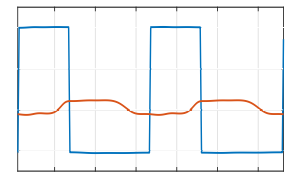

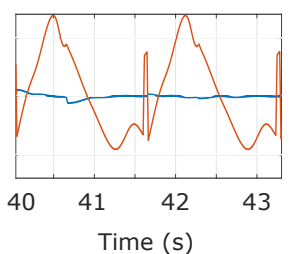

(d)

Figure 3. Simulation results with different frequency combinations (a) $\omega_{s 1}=1 \mathrm{~Hz}$ and $\omega_{s 2}=1 \mathrm{~Hz}$. (b) $\omega_{s 1}=1.2 \mathrm{~Hz}$ and $\omega_{s 2}=1 \mathrm{~Hz}$. (c) $\omega_{s 1}=1.4 \mathrm{~Hz}$ and $\omega_{s 2}=1 \mathrm{~Hz}$. (d) $\omega_{s 1}=1.6 \mathrm{~Hz}$ and $\omega_{s 2}=1 \mathrm{~Hz}$. $S_{1}$ is the simulated signal; the black solid line represents the signal with $\omega_{s 1}$ and the black dotted line represents the signal with $\omega_{s 2} \cdot \varphi_{s}$ is the phase of the simulated signal. $\omega_{S}$ is the estimated frequency of the AO (Red) and the proposed method (Blue). $e_{s}$ is the phase estimation error of the AO (Red) and the proposed method (Blue).

\subsection{Simulated Scenario 2}

When an elderly person uses a walker, the amplitude of the slower oscillation related to walking varies over time owing to their deteriorated abilities. Although this variation does not cause estimation failure, the accuracy can severely decrease. Moreover, the estimated gait phase may decrease instead of increase, leading to failure in gait-phasebased control. Therefore, we simulated a signal to verify the performance of the proposed adaptive rule under a sudden change in amplitude. The simulated signal $S_{2}$ used for the simulation (Figure 4) is given by

$$
S_{2}(t)=\left\{\begin{array}{ccc}
50 \times \sin (2 \times \pi \times t)-25 & \text { for } & 20.5<t<21.5 \\
75 \times \sin (2 \times \pi \times t) & \text { for } & \text { others }
\end{array}\right.
$$

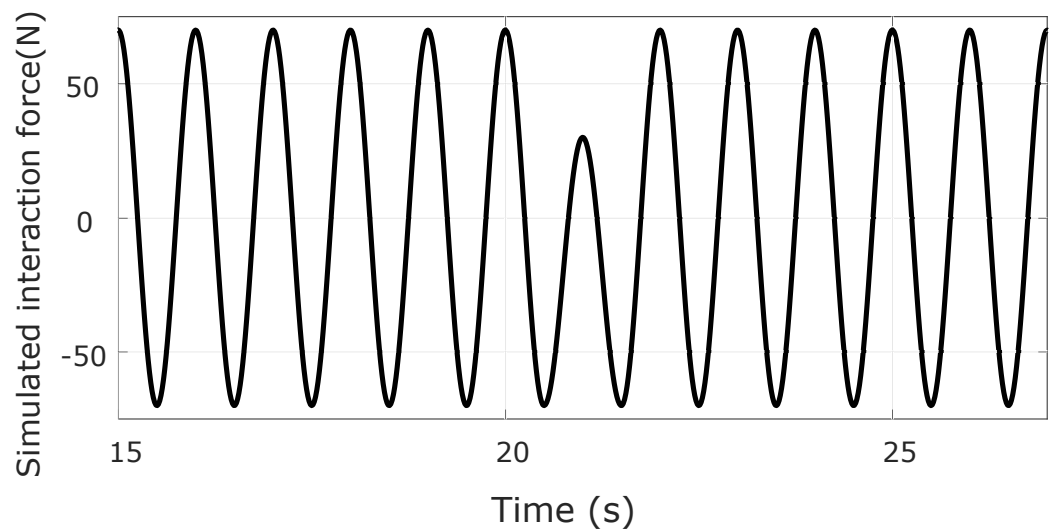

Figure 4. Simulated signal with transient changing amplitude. 
Figure 5 shows the phase estimation results when the amplitude changed suddenly. The transient change in the input signal causes a large perturbation in phase estimation. Specifically, the phase estimation drops for approximately $0.5 \mathrm{~s}$ and increases again instead of monotonically increasing as expected. The behavior in the decreasing phase considerably degrades the estimation accuracy, possibly leading to the failure of gait-phase-based control. On the contrary, the estimation of the proposed method managed to mitigate the influence of the transient change by adaptively reducing the value of $\sigma$ as shown in Figure 6. In practice, the amplitude of the input signal changes frequently, and its influence needs to be controlled.

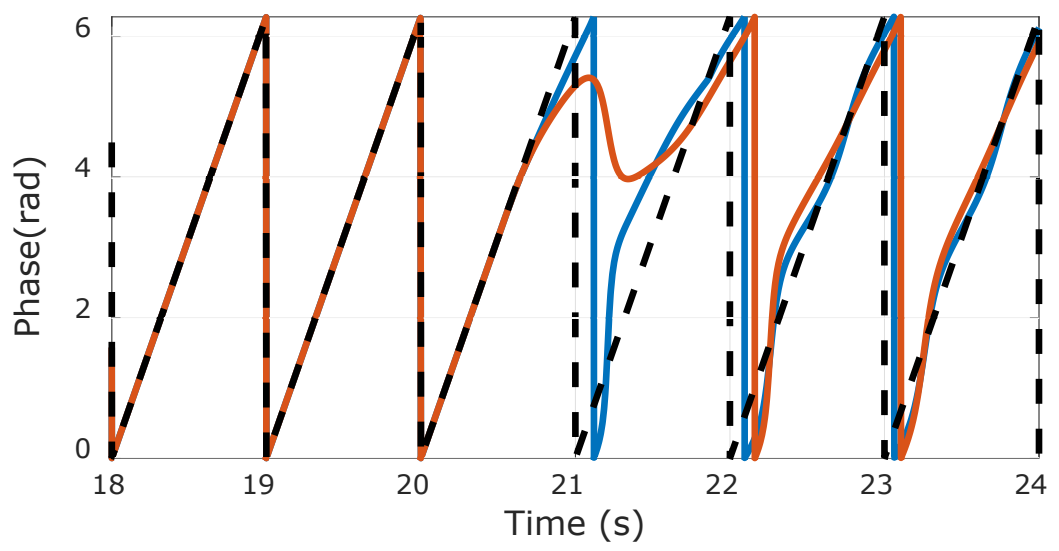

Figure 5. Phase estimation results. The black line is the reference phase, the blue line is the estimated phase of the proposed method, and the red line is the estimated phase of the AO.

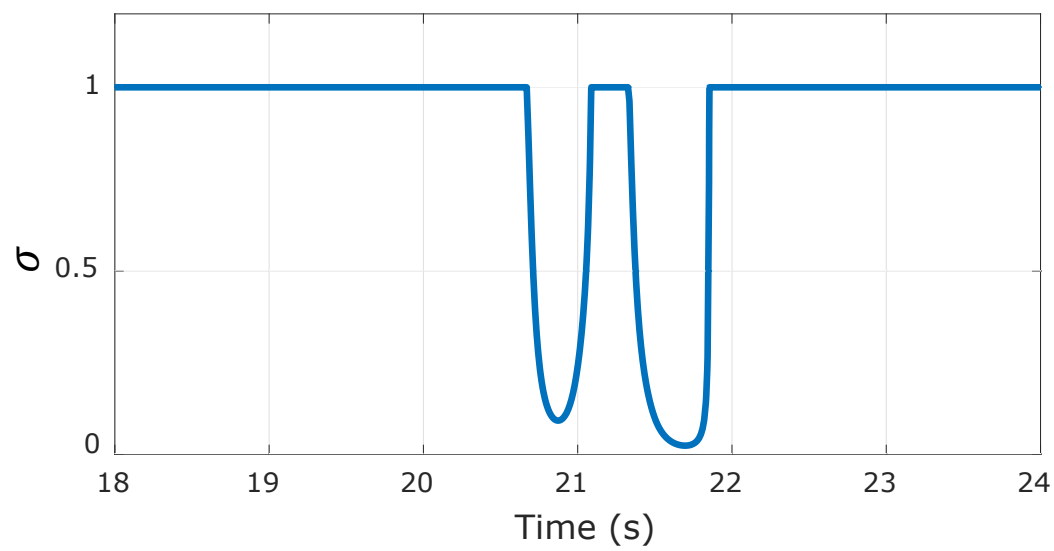

Figure 6. The value of $\sigma$.

\section{Experiment}

\subsection{Experiment Platform}

The experimental robotic walker shown in Figure 7 consists of a mobile platform with four casters (two motor-actuated rear casters and two front passive omnidirectional casters). The walker was developed at the National Innovation Center, Nagoya University, Japan [5].

A six-axis force sensor (WEF-6A500-10-RCD-B, WACOH-TECH, Toyama, Japan) mounted under the handle was used to measure and digitize the user-walker interaction force. A computer was used to estimate the user gait phase from the measured interaction force using inhouse software. The sampling rate of the system was $62.5 \mathrm{~Hz}$.

A pair of pressure sensors (FSR-406, Interlink Electronics, Irvine, CA, USA) was placed under the heels of users to detect the HS instant, as shown in Figure 7. The corresponding measurements were used as the reference for evaluating the accuracy of the proposed method. Both pressure sensors were synchronized with the walker system at a sampling rate of $62.5 \mathrm{~Hz}$. 


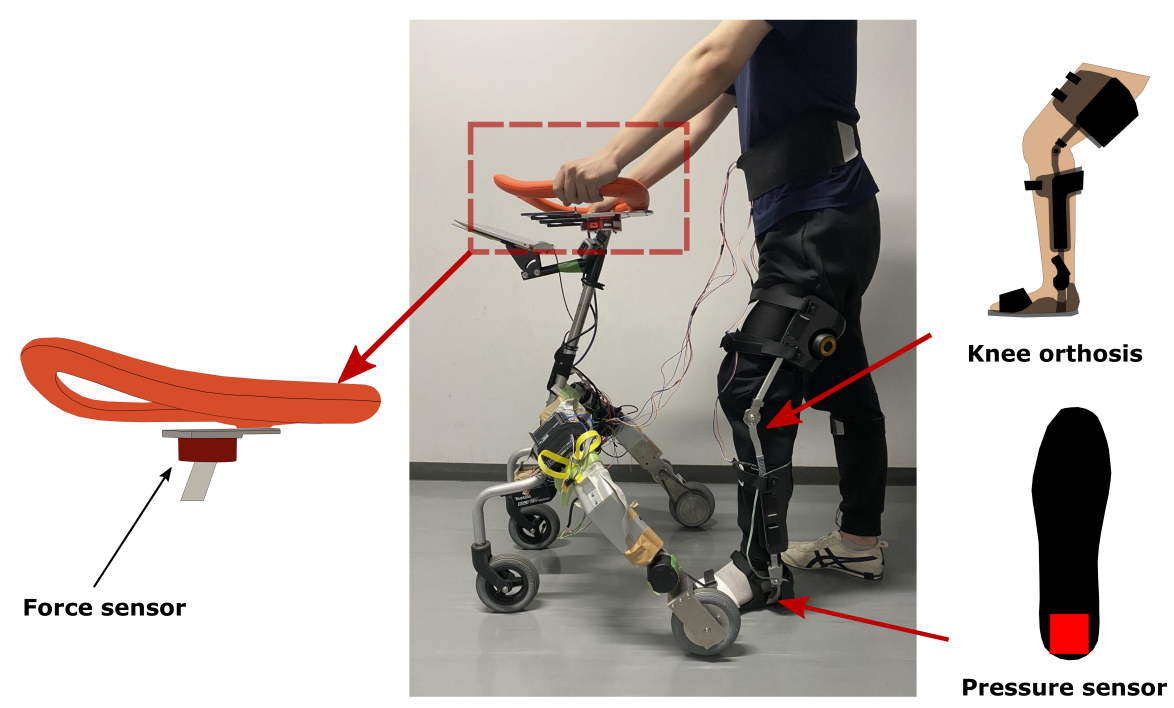

Figure 7. Walker used in experiment.

\subsection{Experiment Protocol}

Nine subjects were recruited for the experiments to evaluate the performance of the proposed gait phase estimation method. The participants were young healthy male students (age: $25.2 \pm 2.0$, height: $176.1 \pm 4.6 \mathrm{~cm}$, bodyweight: $66.5 \pm 6.8 \mathrm{~kg}$ ) from Nagoya University. Before the experiments, the participants were informed of the experimental protocol and signed a consent form. The study was approved by the ethics committee of the Department of Mechanical Systems Engineering, Nagoya University (approval number: 21-10).

The experiment was conducted in two scenarios. (1) Every participant was instructed to walk using a walker along a corridor of $30 \mathrm{~m}$ at a self-selected pace. (2) Every participant repeated the experiment for Scenario 1 while wearing a knee orthosis (GAIT INNOVATION Dial Lock, Pacific Supply, Osaka, Japan). The experiments for the two scenarios were repeated four times per participant. Figure 7 shows the knee orthosis used to restrict the left knee extension to a maximum of $30^{\circ}$ to elicit asymmetric gait patterns $[28,29]$. For the height of the handle, we firstly set the level at half of their body height, and then we slightly adjusted the height based on the feedback from the user individually for better adjustment.

\subsection{Data Analysis}

In this study, we consider a normalized gait phase as the reference gait phase, which linearly increases from 0 to $2 \pi$ in one step cycle. The phase of one step instead of one stride was used to evaluate the estimation performance because we considered asymmetric gait. The instant when the force between the participant's heel and the ground sharply increased was set as the HS instant. The instant when the estimated gait phase reached $2 \pi$ was considered as the estimated HS instant. Figure 8 shows the difference between the reference and estimated gait phases (i.e., estimation error), and the average root mean square error (RMSE) across the experiment was used to quantify the accuracy of gait phase estimation. The HS error determines the estimation performance because the HS is the most important gait event. During the experiment, gait phase estimation failed to detect HS in some cases, as shown in Figure 9. The HS misdetections were discarded to calculate the estimation error. The number of misdetections was recorded for evaluation. For all the trials, the first five strides were discarded to exclude the synchronization period between the $\mathrm{AO}$ and interaction force, and the other steps were considered for evaluation.

We also compared the proposed method with the direct use of an $\mathrm{AO}$ to determine the effectiveness of the proposed adaptive rule and switching mechanism for estimation. The results of the proposed method were obtained in real time, while those of the AO 
were obtained offline in MathWorks MATLAB from the same experimental data and same parameter settings ( $\mu=1, \epsilon=0.1, \sigma=1, \delta=1$, and $\lambda=0.3$ ). All of the parameters are tuned by simulations in Matlab/Simulink and confirmed in the preliminary stage of the experiment.

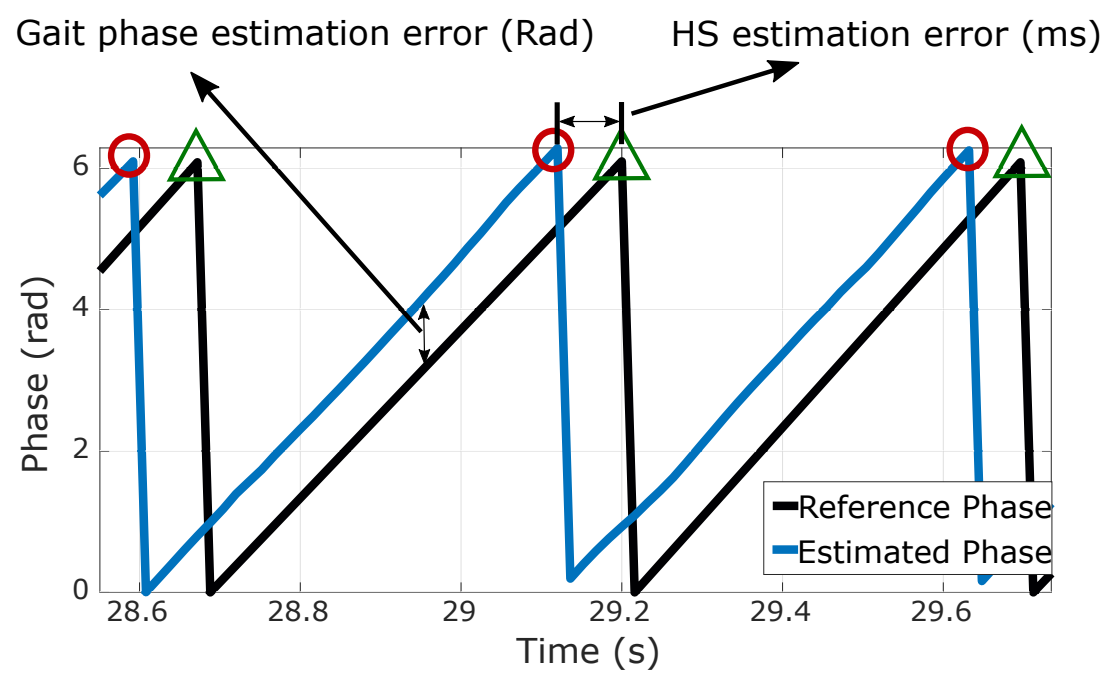

Figure 8. Gait phase estimation error and Heel Strike (HS) estimation error (red circles = estimated HS; green triangles = HS detected by pressure sensor) .

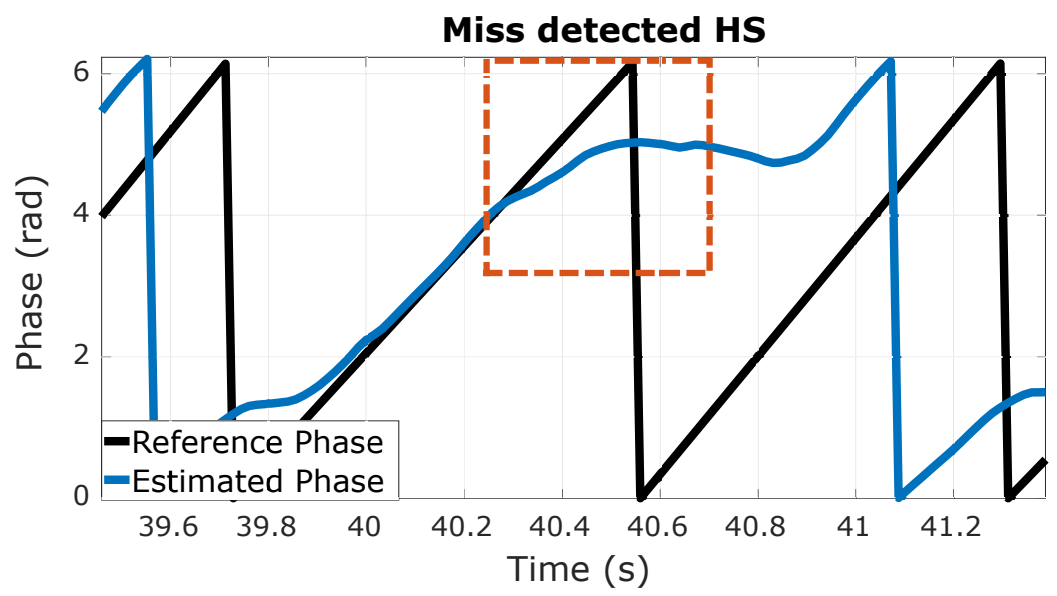

Figure 9. HS misdetections.

A one-way analysis of variance was applied to analyze significant differences in the estimation of HS instants. Pearson's chi-squared test is applied to evaluate the significant difference in the number of misdetections. The threshold for the significance was set to 0.01 .

All the analyses were implemented in MATLAB 2019a.

\subsection{Results}

The results for Scenario 1 are listed in Table 1. The estimation results are similar for the conventional AO and proposed method. Nevertheless, the AO provided $35 \mathrm{HS}$ misdetections over the 36 trials (2884 steps), while the proposed method only provided four HS misdetections. There was a significant difference in the number of misdetections between the two methods $(p<0.01)$. 
Table 1. Estimation performance when participants walked without knee restriction.

\begin{tabular}{cccc}
\hline Name of Method & Gait Phase Estimation Error (RMSE) & HS Estimation Error & No. HS Misdetections (Ratio *) \\
\hline AO & $0.774 \mathrm{rad}$ & $54.16 \pm 53.28 \mathrm{~ms}$ & $35(1.21 \%)$ \\
Proposed method & $0.691 \mathrm{rad}$ & $55.75 \pm 56.55 \mathrm{~ms}$ & $4(0.14 \%)$ \\
\hline
\end{tabular}

* Percentage for HS misdetections of HSs in all trials.

Table 2 shows that the difference between the two methods became much larger when the participants walked with the knee restriction. The consecutive step times were different from each other: the step time from left HS to right HS was $0.766 \pm 0.143 \mathrm{~s}$, while the step time from right HS to left HS was $0.642 \pm 0.108 \mathrm{~s}$. In addition, the accuracy of HS instant estimation degraded significantly for both methods because the interaction force contained more variations and disturbance due to the use of the knee restriction. There were significant differences $(p<0.01)$ between the HS estimation errors and the number of the misdetections of the two methods. Moreover, the number of HS misdetections provided by the conventional $\mathrm{AO}$ (105 misdetections) was much larger than that provided by the proposed method (11 misdetections) for 3174 steps during the experiment with knee movement restriction.

Table 2. Estimation performance when participants walked with knee restriction.

\begin{tabular}{cccc}
\hline Name of Method & Gait Phase Estimation Error (RMSE) & HS Estimation Error & No. HS Misdetections (Ratio *) \\
\hline AO & $0.889 \mathrm{rad}$ & $80.61 \pm 70.39 \mathrm{~ms}$ & $105(3.31 \%)$ \\
Proposed method & $0.722 \mathrm{rad}$ & $75.79 \pm 66.69 \mathrm{~ms}$ & $11(0.35 \%)$ \\
\hline
\end{tabular}

* Percentage for HS misdetections of HSs in all trials.

\section{Discussion}

We propose a gait phase estimation method based on AOs and the user-walker interaction force. First, we use a BMFLC to extract the slow oscillation component that reflects walking in the interaction force. Then, we use AOs for phase estimation. In addition, a switching mechanism and a parameter-adaptive rule improve the accuracy and robustness of the AOs under rapid changes in amplitude and frequency of the input gait signal. The switching mechanism allows two AOs to track the frequencies of consecutive steps to account for asymmetric gait. As shown in the simulation, with the increase in the frequency difference between the two legs, the estimation error keeps increasing constantly for the single $\mathrm{AO}$. When the frequencies are $1.4 \mathrm{~Hz}$ and $1 \mathrm{~Hz}$, the estimation error reaches $0.7 \mathrm{rad}$, which is about approximately $10 \%$ of a step time (5\% of a stride time if the gait is symmetric), while the estimation error is much lower with the proposed switching mechanism. An estimation error of $5 \%$ of the gait cycle is not substantial; however, the simulated signal is a pure sinusoidal, while the interaction force contains variations and disturbances, which can deteriorate the estimation accuracy. If the estimation error is over $10 \%$ of the gait cycle, it leads to the failure of the control algorithm when the target gait phases are those with short durations, such as the bodyweight acceptance, which lasts for approximately $10 \%$ of the gait cycle [30]. By adopting the proposed adaptive rule, parameter $\sigma$ is adapted based on the difference in estimated frequency $\omega$ and actual change rate of phase $\dot{\varphi}$. The proposed adaptive rule considers that the gait phase does not change drastically during walking and is applied when the difference is large, thus maintaining a phase increase of at least $(1-\lambda)$ of the expected frequency. Nevertheless, this method may decelerate the initial $\mathrm{AO}$ synchronization when the phase difference between the estimated and true gait phases is large [19,31]. However, the deterioration of synchronization is suppressed afterward, and there is no substantial influence on the overall estimation accuracy.

In the experiments, the phase estimation accuracy of scenario 1 (without knee restriction) was better than that of scenario 2 (with knee restriction) because the interaction force contains more disturbances and variations under the limitations imposed by the knee 
orthosis. In fact, a restricted knee movement hampers the coordinative movement of the trunk during walking [32], eventually altering the interaction force during walking. The estimation performance of the proposed method and a single $\mathrm{AO}$ is similar without the restriction. As the participants in this study were young and healthy, their gait patterns were symmetric and the interaction force contained few variations and noise, promoting accurate estimation. In contrast, the proposed method showed higher accuracy than $\mathrm{AO}$ when the participants walked with a knee orthosis, which elicited asymmetric gait due to the restricted knee movement. For these subjects, the RMSE of phase estimation provided by the proposed method was lower than that without the adaptive rule and switching mechanism.

The switching mechanism improved the estimation accuracy by using two separate states to track the step timing of the two legs. Although the difference in HS estimation error between the proposed method and single $\mathrm{AO}$ was significant, the improvement in the estimation accuracy was not very large (reduction by 6.25\%). However, over $100 \mathrm{HS}$ misdetections occurred using the conventional AO, whereas most HSs were captured by the proposed method. With a smaller $\sigma$, the single AO method can achieve fewer misdetections of heel strikes. However, it takes a longer time for the AO to re-synchronize with the input signal when the characteristics of the input signal change. This will eventually deteriorate the estimation accuracy. Although the proposed method successfully detected the HSs, the estimation accuracy was degraded when the orthosis was used. These differences in estimation are likely much larger if the HSs are neglected.

The HS estimation error was also compared with offline gait event estimation based on the user-walker interaction force proposed in [10]. This method uses a Butterworth bandpass filter to suppress noise, and the peak timing of the interaction force is considered as the HS instant. Its estimation accuracy was $46.75 \pm 50.60 \mathrm{~ms}$, outperforming the results of our proposal. However, the HS estimation error of the proposed method in scenario 1 was $54.16 \pm 53.28 \mathrm{~ms}$, being comparable to the results of Abellenas's method. This is a suitable result considering that the results were obtained offline by the method in [10], while the proposed method performs real-time estimation. Abellenas's paper does not report any misdetection of HS, but the misdetection of HS is mentioned in other similar research studies $[9,33]$ while none of them uses the misdetection of HS to evaluate the performance of their estimation methods.

The adaptive rule for $\sigma$ improves not only the estimation performance but also the robustness. Using the proposed method, the number of HS misdetections decreased considerably from 105 to 11 . In the few cases in which the proposed method provided HS misdetections, the interaction force did not reflect the change in the gait phase. For example, Figure 10 shows the filtered interaction force for an HS misdetection provided by the proposed method. The HS instant is supposed to coincide with the peak timing of the interaction force, but the peak in the red circle is very small, leading to a failed HS detection. This is a limitation of the proposed method because its results rely on the interaction force. In addition, the gait phase can be indirectly affected by elbow movements or posture changes during walking.

If a heel strike is not detected by the proposed method, the AO that kept track of the left steps will turn to track of the right steps while the AO that kept track of the right steps will turn to track of the left steps. It will take several steps for the estimator to re-synchronize with the input signal, which will deteriorate the performance of estimation in a short period. However, the phase estimation error will diminish rapidly during the re-synchronization period. Furthermore, the misdetections barely occur for the proposed method even when the user's knee movement is restricted (11 misdetections for about 3000 steps), and the phase estimation error after the 11 misdetections decreases rapidly due to the choice of a $\epsilon$ with a large value to increase the converge rate of $\omega$. Therefore, it is not a big issue for the proposed phase estimation method. Moreover, we will improve the proposed method by making the best use of the interaction force/torque between the walker and a user to determine the left/right heel strikes in real time. During walking, 
the interaction force (vertical direction) at the corresponding side grows, whereas the interaction force on the opposite side decreases, as demonstrated in [33]. In this way, the proposed method can keep track of left steps with one AO and keep track of right steps with another AO.

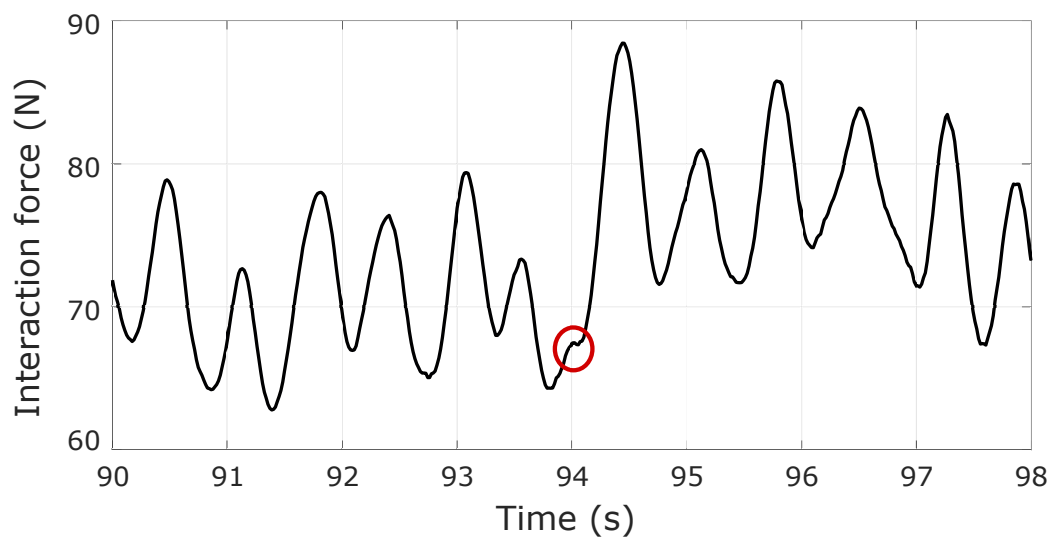

Figure 10. Interaction force under HS misdetection (red circle).

As the proposed method relies on the user-walker interaction force for gait phase estimation, it may fail to detect the HS instant and gait phase if the interaction force contains several unrelated fluctuations. In the experiment, 11 HS misdetections occurred when the knee movement was restricted, which mostly negligible because over 3000 steps were processed. HS misdetection is mainly caused by elbow movement and posture changes during walking, and the estimation performance may degrade for elderly people, who often present deteriorated upper and lower limb strength.

In the experiments, all nine participants are young healthy males, and a knee orthosis is used to elicit asymmetric gait. For elderly people, there can be a larger amount of noise and variations in the amplitude and frequency of interaction forces due to their deteriorating upper and lower limb strength. The noise and variations can degrade the estimation accuracy of the proposed method. Although a knee orthosis can resemble the gait patterns of people who suffer from joint contracture, the gait patterns of people with motor impairments are more diverse. For instance, some stroke survivors suffer from hemiplegia, which not only leads to an asymmetric gait but also damages their upper limb muscles and trunk muscles on the affected side. The interaction force between a user suffering from a severe stroke and a walker can be different from that between a healthy user and a walker. It may change the correlation between the interaction force and the gait phase that is used for gait phase estimation. The changed correlation may make it less reliable for the proposed method to estimate the gait phase successfully.

On the contrary, gender does not seem to be a factor that will substantially influence the gait phase estimation. The differences between male and female users are the height and the body weight, and female users are normally shorter and lighter. The height of the handle of the walker is adjustable, and it can be adjusted to fit people with different heights. While the lighter body weight can lead to a smaller amplitude in the interaction force, it is not a major issue since the AO can adapt its amplitude to synchronize with the interaction force with different amplitudes. We will confirm this by evaluating the performance with both male and female subjects in our future work.

All experiments were conducted for straight walking, and it is expected that performance in curve walking should be slightly worse than that in the straight line walking, as was presented in Alwan's research [9]. There will be an additional component in the interaction force that represents the user's intention to change the direction of walking, which is a disturbance to the gait phase estimation.

The real-time gait phase estimation method will be used to enhance the performance of lower limb physical training for elderly people. By using the proposed real-time gait phase estimation method, we tried to exert different levels of resistance to young healthy 
subjects at different gait phases to achieve better training outcomes for certain lower limb muscles [11]. As an example, we increased the level of resistance to a user at $10 \%$ to $50 \%$ of a gait cycle to train the muscle of gastrocnemius. We assume that the gait patterns of people with mild motor impairments (elderly people and patients) are similar to those of young healthy people. However, there are two limitations: (1) the gait pattern may change due to the resistance exerted to the user or the physical conditions of the user, just as stroke can alter the gait pattern significantly [34]; (2) the gait patterns of elderly people or people with motor impairments are inconsistent due to the deteriorated walking ability. Furthermore, the proposed gait phase estimation method can only estimate the percentage of a gait cycle rather than identifying the phases of a gait such as loading response, mid-stance, or pre-swing. The effectiveness of the physical training can degrade if the resistance is not applied at desired gait phases.

\section{Conclusions and Future Work}

We propose a robust real-time gait phase estimation method that considers asymmetric gait patterns and relies on the user-walker interaction force. In addition, the proposed method uses two AOs (Adaptive Oscillators) that are alternately activated by a switching mechanism. The switching mechanism enables accurate gait phase estimation even for people presenting asymmetric gait. Furthermore, an adaptive rule for an $\mathrm{AO}$ parameter improves the transient performance under drastic amplitude changes in the input gait signal. The performance of the proposed method and its underlying techniques were evaluated in simulations. The proposed techniques can improve the accuracy of gait phase estimation for asymmetric gait and sudden amplitude changes. Then, we experimentally validated the proposed method, and we found that (1) the proposed method achieves higher accuracy and robustness than the conventional AO, especially under asymmetric gait elicited by a knee orthosis; (2) the proposed method can estimate the HS instant with high accuracy ( $54.16 \pm 53.28 \mathrm{~ms})$, similar to that of a method for offline gait event detection based on the interaction force $(46.75 \pm 50.60 \mathrm{~ms})$.

From the viewpoint of ethics, we only accepted young healthy subjects in this study. In the future

1. The application of the proposed method for people who suffer from severe motor impairments will be further investigated;

2. The application of the proposed method for actual wheel walker users under different conditions (straight and curve walking) will be further investigated;

3. The interaction forces to be detected at both sides will be used to minimize the influence of misdetections of HSs (heel strikes).

as were presented in the discussion section.

Moreover, we will apply the proposed method for improving the effectiveness of physical training for elderly people with a smart walker.

Author Contributions: Methodology, Y.Y. and P.L.; writing original draft preparation, P.L.; writingreview, X.W., Y.A., and Y.Y.; resources, K.Y. and M.Y.; supervision, Y.Y.; fund acquisition, Y.Y. and K.Y. All authors have read and agreed to the published version of the manuscript.

Funding: This work was supported by Center of Innovation (JPMJCE1317) Program run at Nagoya University, which is driven by Japan Science and Technology Agency. This work was also supported in part by The Research Funding for Longevity Sciences (21-21) from National Center for Geriatrics and Gerontology (NCGG), Japan.

Institutional Review Board Statement: The study was conducted according to the guidelines of the Declaration of Helsinki and approved by the ethics committee of the Department of Mechanical Systems Engineering, Nagoya University (approval number: 21-10).

Informed Consent Statement: Informed consent was obtained from all subjects involved in the study.

Data Availability Statement: Data available on request due to ethical restrictions. 
Acknowledgments: The authors would like to thank Shogo Okamoto for his advice and Izumi Kondo for providing us the opportunity to study in the project of "Development of ICT and robot for the everyday life of older adults".

Conflicts of Interest: The authors declare no conflict of interest.

\author{
Abbreviations \\ The following abbreviations are used in this manuscript: \\ HS Heel Strike \\ AO Adaptive Oscillator \\ BMFLC Band-limited Multiple Fourier Combiner \\ RMSE Root Mean Square Error
}

\title{
References
}

1. Aboutorabi, A.; Arazpour, M.; Bahramizadeh, M.; Hutchins, S.W.; Fadayevatan, R. The effect of aging on gait parameters in able-bodied older subjects: A literature review. Aging Clin. Exp. Res. 2016, 28, 393-405. [CrossRef]

2. Thibaud, M.; Bloch, F.; Tournoux-Facon, C.; Breque, C.; Rigaud, A.S.; Dugue, B.; Kemoun, G. Impact of physical activity and sedentary behaviour on fall risks in older people: A systematic review and meta-analysis of observational studies. Eur. Rev. Aging Phys. Act. 2012, 9, 5-15. [CrossRef]

3. Stevens, J.A.; Powell, K.E.; Smith, S.M.; Wingo, P.A.; Sattin, R.W. Physical activity, functional limitations, and the risk of fall-related fractures in community-dwelling elderly. Ann. Epidemiol. 1997, 7, 54-61. [CrossRef]

4. Page, S.; Saint-Bauzel, L.; Rumeau, P.; Pasqui, V. Smart walkers: An application-oriented review. Robotica 2017, 35, 1243-1262. [CrossRef]

5. Yamada, K.; Yokoya, M.; Yamada, Y. Proposal of walking assistant robot for the elderly which is intended for use in home. IEICE Tech. Rep. 2016, 116, 25-30. (In Japanese)

6. Ballesteros, J.; Urdiales, C.; Martinez, A.B.; Tirado, M. Automatic Assessment of a Rollator-User's Condition during Rehabilitation Using the i-Walker Platform. IEEE Trans. Neural Syst. Rehabil. Eng. 2017, 25, 2009-2017. [CrossRef]

7. Werner, C.; Chalvatzaki, G.; Papageorgious, X.S.; Tzafestas, C.S.; Bauer, J.M.; Hauer, K. Assessing the concurrent validity of a gait analysis system integrated into a smart walker in older adults with gait impairments. Clin. Rehabil. 2019, 33, $1682-1687$. [CrossRef]

8. Alwan, M.; Wasson, G.; Sheth, P.; Ledoux, A.; Huang, C. Passive derivation of basic walker-assisted gait characteristics from measured forces and moments. In Proceedings of the 26th Annual International Conference of the IEEE Engineering in Medicine and Biology Society, San Francisco, CA, USA, 1-5 September 2004. [CrossRef]

9. Alwan, M.; Ledoux, A.; Wasson, G.; Sheth, P.; Huang, C. Basic walker-assisted gait characteristics derived from forces and moments exerted on the walker's handles: Results on normal subjects. Med. Eng. Phys. 2007, 29, 380-389. [CrossRef]

10. Abellanas, A.; Frizera, A.; Ceres, R.; Gallego, J.A. Estimation of gait parameters by measuring upper limb-walker interaction forces. Sensors Actuators Phys. 2010, 162, 276-283. [CrossRef]

11. Li, P.; Yamada, Y.; Wan, X.; Uchiyama, Y.; Sato, W.; Yamada, K.; Yokoya, M. Gait-phase-dependent control using a smart walker for physical training. In Proceedings of the 2019 IEEE 16th International Conference on Rehabilitation Robotics (ICORR), Toronto, ON, Canada, 24-28 June 2019. [CrossRef]

12. Yan, T.; Cempini, M.; Oddo, C.M.; Vitiello, N. Review of assistive strategies in powered lower-limb orthoses and exoskeletons. Robot. Auton. Syst. 2015, 64, 120-136. [CrossRef]

13. Lewis, C.L.; Ferris, D.P. Invariant hip moment pattern while walking with a robotic hip exoskeleton. J. Biomech. 2011, 44, 789-793. [CrossRef]

14. Zhang, Y.; Nolan, K.J.; Zanotto, D. Oscillator-based transparent control of an active/semiactive ankle-foot orthosis. IEEE Robot. Autom. Lett. 2011, 4, 247-253. [CrossRef]

15. Ronsse, R.; Lenzi, T.; Vitiello, N.; Koopman, B.; van Asseldonk, E.; De Rossi, S.M.; van den Kieboom, J.; van der Kooij, H.; Carrozza, M.C.; Ijspeert, A.J. Oscillator-based assistance of cyclical movements: Model-based and model-free approaches. Med. Biol. Eng. Comput. 2011, 49, 1173-1185. [CrossRef]

16. Righetti, L.; Buchli, J.; Ijspeert, A.J. Dynamic Hebbian learning in adaptive frequency oscillators. Phys. D Nonlinear Phenom. 2006, 216, 269-281. [CrossRef]

17. Righetti, L.; Buchli, J.; Ijspeert, A.J. Adaptive Frequency Oscillators and Applications. Open Cybern. Syst. J. 2009, 3, 64-69. [CrossRef]

18. Yan, T.; Parri, A.; Ruiz Garate, V.; Cempini, M.; Ronsse, R.; Vitiello, N. An oscillator-based smooth real-time estimate of gait phase for wearable robotics. Auton. Robot. 2017, 41, 759-774. [CrossRef]

19. Nachstedt, T.; Tetzlaff, C.; Manoonpong, P. Fast Dynamical Coupling Enhances Frequency Adaptation of Oscillators for Robotic Locomotion Control. Front. Neurorobot. 2017, 11-14. [CrossRef] 
20. Zhao, J.; Iwasaki, T. Orbital Stability Analysis for Perturbed Nonlinear Systems and Natural Entrainment via Adaptive AndronovHopf Oscillator. IEEE Trans. Autom. Control 2020, 65, 87-101. [CrossRef]

21. Patterson, K.K.; Parafianowicz, I.; Danells, C.J.; Closson, V.; Verrier, M.C.; Staines, W.R.; Black, S.E.; McIlroy, W.E. Gait asymmetry in community-ambulating stroke survivors. Arch. Phys. Med. Rehabil. 2008, 89, 304-310. [CrossRef]

22. Frizera Neto, A.; Gallego, J.A.; Rocon, E.; Pons, J.L.; Ceres, R. Extraction of user's navigation commands from upper body force interaction in walker assisted gait. Biomed. Eng. Online 2010, 9, 37. [CrossRef]

23. Veluvolu, K.C.; Wang, Y.; Kavuri, S.S. Adaptive estimation of EEG-rhythms for optimal band identification in BCI. J. Neurosci. Methods 2012, 203, 163-172. [CrossRef] [PubMed]

24. Xiao, F.; Gao, Y.; Wang, S.; Zhao, J. Prediction of pathological tremor using adaptive multiple oscillators linear combiner. Biomed. Signal Process. Control 2016, 27, 77-86. [CrossRef]

25. Narendra, K.S.; Balakrishnan, J. Improving Transient Response of Adaptive Control Systems using Multiple Models and Switching. IEEE Trans. Autom. Control 1994, 39, 1861-1866. [CrossRef]

26. Branicky, M.S. Lyapunov functions and other analysis tools for switched and hybrid systems. IEEE Trans. Autom. Control 1998, 43, 475-482. [CrossRef]

27. Westervelt, E.R.; Grizzle, J.W.; Canudas de Wit, C. Switching and PI control of walking motions of planar biped walkers. IEEE Trans. Autom. Control 2002, 48, 308-312. [CrossRef]

28. Cerny, K.; Perry, J.; Walker, J.M. Adaptations during the stance phase of gait for simulated flexion contractures at the knee. Orthopedics 1994, 17, 501-512. [CrossRef]

29. Harato, K.; Nagura, T.; Matsumoto, H.; Otani, T.; Toyama, Y.; Suda, Y. A gait analysis of simulated knee flexion contracture to elucidate knee-spine syndrome. Gait Posture 2008, 28, 687-692. [CrossRef]

30. Perry, J.; Burnfield, J.M. Gait Analysis: Normal and Pathological Function, 2nd ed.; Slack Inc.: New York, NY, USA, 2010.

31. Karimi Ghartemani, M.; Khajehoddin, S.A.; Jain, P.K.; Bakhshai, A. Problems of Startup and Phase Jumps in PLL Systems. IEEE Trans. Power Electron. 2012, 27, 1830-1838. [CrossRef]

32. Yang, S.; Zhang, J.T.; Novak, A.C.; Brouwer, B.; Li, Q. Estimation of spatio-temporal parameters for post-stroke hemiparetic gait using inertial sensors. Gait Psoture 2013, 37, 354-358. [CrossRef]

33. Ballesteros, J.; Urdiales, C.; Martinez, A.B.; van Dieën, J.H. On Gait Analysis Estimation Errors Using Force Sensors on a Smart Rollator. Sensors 2016, 16, 1896. [CrossRef]

34. Goldie, P.A.; Matyas, T.A.; Evans, O.M. Gait after stroke: Initial deficit and changes in temporal patterns for each gait phase. Arch. Phys. Med. Rehabil. 2001, 82, 1057-1065. [CrossRef] [PubMed] 